\title{
The Mighty Microbiota: Regulator of the Human Body
}

\section{P D Gupta}

Former, Director Grade Scientist, Centre for Cellular and Molecular Biology, Hyderabad, India.

*Corresponding Author: P D Gupta, Former, Director Grade Scientist, Centre for Cellular and Molecular Biology, Hyderabad, India.

Received date: June 02, 2021; Accepted date: June 26, 2021; Published date: June 25, 2021

Citation: P D Gupta. (2021) The Mighty Microbiota: Regulator of the Human Body. Clinical Research and Clinical Trials. 3(5); DOI: $10.31579 / 2693-4779 / 048$

Copyright: () 2021 P D Gupta. This is an open access article distributed under the Creative Commons Attribution License, which permits unrestricted use, distribution, and reproduction in any medium, provided the original work is properly cited.

\begin{abstract}
Microbiota is a life line for human being, however if the balance in interspecies of microbiota is disturb, it can cause not only serious diseases but can kill also. Collectively the microbiotal species act as epigenetic factor for humans. First exposure to microbiota is in utero. The whole health programming of the individuals stars even before birth. C-section or fed formula fed babies are immunologically weaker than that of normal delivered and beast fed babies. For the lifelong good health of babies, Mothers should opt for vaginal delivery and breastfeeding for healthy newborn
\end{abstract}

Keywords: microbiota; serious diseases; epigenetic

Microbiota is found in all most all organs in the human body and body fluids. This group of microorganism is a conglomeration of bacteria, fungi, protozoans and viruses. Earlier, considering them as harmful creatures, Luis Pasture designed antibodies to kill them. It was a big epoch in the history of medicine; many inflamed lives were saved [1]. Later it was found that only some of them are harmful and can cause infections, but many species of this group of organisms are supportive and beneficial and thus are essential for the body's wellbeing and good health [2]. Some microorganisms that live on/in humans are commensal. Certain microorganisms perform tasks that are known to be useful to the human host however the mechanism of action of most of them is not well understood. Those that are expected to be present, and that under normal circumstances do not cause disease, are sometimes deemed as normal microbiota. Sometimes, the microbiota activates the immune system to get rid of dangerous bacteria and prevent even diseases caused by bacteria and/or viruses. There are some organs where if there is no microbiota, it is difficult to survive, however one can live without, if no microbiota present in our spleen, gall bladder, tonsils and appendix. But we wouldn't survive long without our gut microbes. Intriguingly, no two individual's microbiota is the same; in this respect we are all unique. And more than ever, we're finding out just how important these microbes are for us [3].

\section{Human and bacterial cells}

Using mathematical modelling a team of researchers from Greece, Italy, and Spain in 2013 published an estimation of the number of cells in the human body. This paper put the number of cells at $37.2+/-0.81$ trillion [4].

Later in 2016, Ron Milo [5] an associate professor at the Weizman Institute of Science, Rehovot, Israel, and colleagues published an update. In fact, they performed two different calculations. The first one estimates the number of cells in a 100-kilogram male using an average cell volume of between 1,000 and 10,000 cubic micrometers. This gave them a "back of the envelope estimate" in a range of 30 to 40 trillion cells.

Next, they calculated the actual cell number of the five most common cell types in an average adult male, which account for 97 percent of the cells in the body. This led them to an estimate of 30 trillion cells, of which red blood cells make up 84 percent.

But human cells are not the only cells in our bodies. Although previous studies have estimated that there are 10 times as many bacteria in our bodies than human cells, Prof. Milo and colleagues revised this number to be around 38 trillion. Interestingly, although large in number, bacteria are much smaller than human cells, and they actually make up only 200 grams of total body mass [5]. But with nearly equal numbers of cells in our bodies, one could argue that we are as much bacteria as we are human, bringing the total number up to around 70 trillion.

\section{How do we get microbiota?}

Neonates generally acquire intestinal microbial communities within the first week of life with relative equilibrium reached within the first year. Infants delivered via caesarean birth have higher rates of colonization by environmentally acquired microbes such as Clostridium difficile and Klebsiella and Enterobacter species, and have enhanced colonization of the neonatal oral cavity and intestine by components of the skin microbiome. In addition, the Ureaplasma species as well as Mycoplasma hominis are associated with preterm birth.

All new born babies get their microbiota from mothers at the time of birth though some scientists believe that there is an exposure of microbiota to the preterm baby in order to felicitate to develop immune system. The delivery method influences infants' microbial makeup. University of Edinburgh-led team collected fecal samples at 10 time points from 46 babies born by Caesarean section and 74 babies born vaginally for 
analysis [6] The two groups of infants had different microbiomes,. It notes that mothers were only given antibiotics after having given birth and that there were still differences between babies that were bottle-fed, indicating that antibiotic exposure through breast milk couldn't account for the effect. Infants born via Caesarean section have more bacteria that are considered "bad" and fewer that are considered "good" resident among their gut microbiota, [7] which was Completed in 2012, characterized the microbiome composition of multiple body sites in healthy individuals of different ethnicities located in two separate cities (St. Louis, Missouri and Houston, Texas) in the United States. This multicenter effort showed that bacterial diversity, niche specificity, and microbial gene carriage patterns far exceeded what was initially suspected [8-12].

The Human Microbiome Project (HMP) * The Human Microbiome Project was a United States National Institutes of Health research initiative to improve understanding of the microbial flora involved in human health and disease. Launched in 2007, the first phase focused on identifying and characterizing human microbial flora. Established: 2007, Start date: 2008 Planned completion date: 2013 but completed in 2012

\section{Omnipresent Microbiota}

Humans acquire these bacteria during birth (especially vaginal delivery) and the first years of life, and they live with us throughout our lives. Somewhere between 300 and 1000 different species live in the gut, with most estimates at about 500. However, it is probable that $99 \%$ of the bacteria come from about 30 or 40 species, with Faecalibacterium prausnitzii being the most common species in healthy adults. There is a greater diversity of bacteria living on the human forearm than on any other part of the body, according to a new study [13] on average, 44 different types of bacteria reside on the forearm, compared with 19 species living behind the ear, says the study by the National Human Genome Research Institute in the US.

Anatomically microbiota is found on/in the human body sites are as follow:

\section{A. Anatomical areas}

Skin (especially the moist areas, such as the groin and between the toes), Conjunctiva respiratory tract (particularly the nose), Gastrointestinal tract(primarily the mouth and the colon), Urethra and bladder( urinary tract), Vagina, Placenta, Uterus, Oral cavity/ mucosa, Lung, Mammary glands, Ovarian follicles, Placenta,

\section{B. Body Fluids}

\section{Regulatory factors for microbiota}

Gut: Conceptually, we should view these microbes as a newly discovered organ, weighing slightly more than our brains and nearly as vital. Gastrointestinal tract microbiota seems to have a major influence on microbiota present in the body elsewhere and diet (14). These microbes mostly live in the lower intestine (the colon) and outnumber all the other cells in the body put together. And they are regulated by various factors such as body weight, propensity to illness, immune system, appetite and mood.

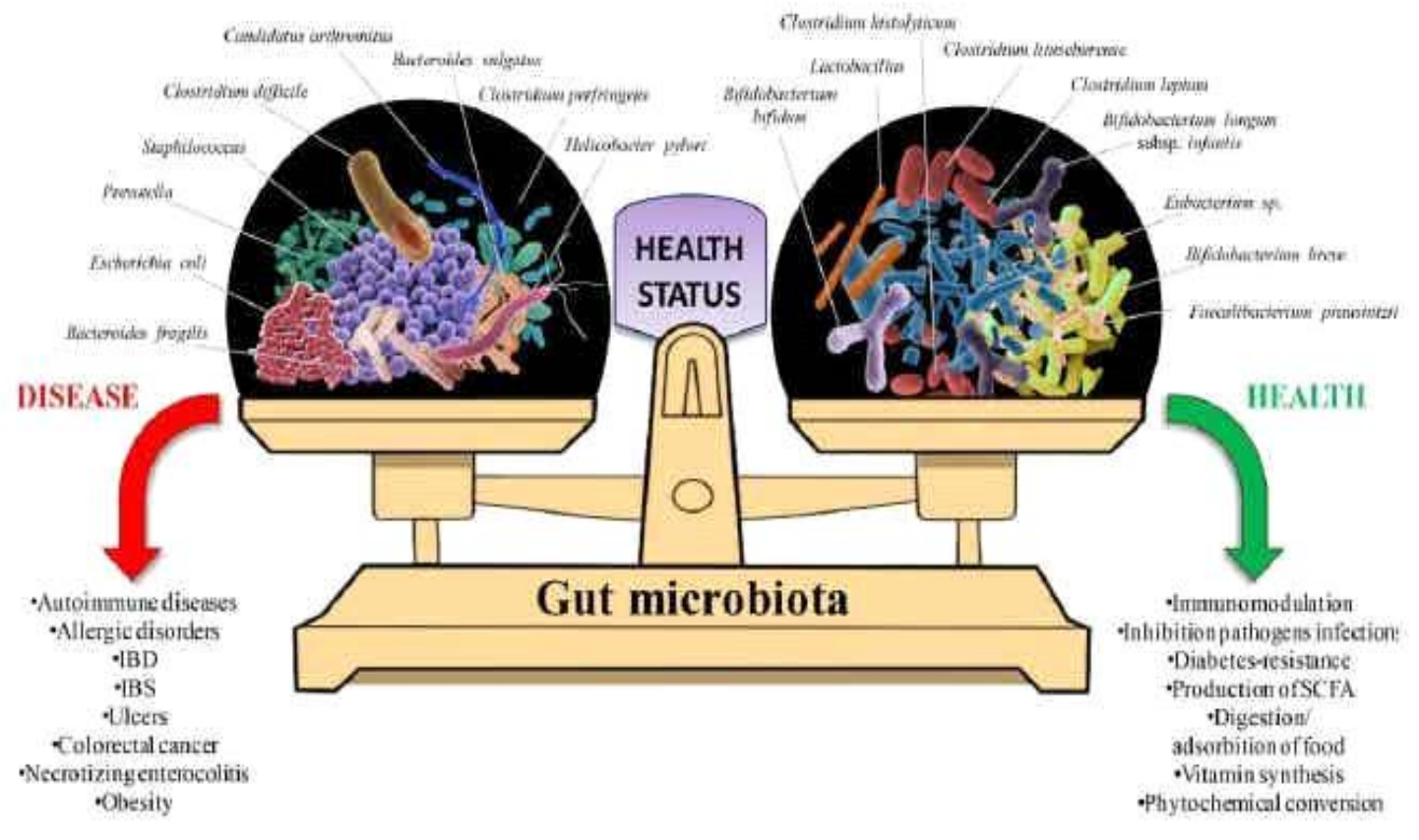

Figure 1: Schematic representation of the functional roles of key members of the human gut microbiota in health and disease. IBD, inflammatory bowel disease; IBS, irritable bowel syndrome; SCFA, short-chain fatty acid (after, Milani et al., 2016, ref. 14).

According to recent research, the richer and more diverse the community of gut microbes are, the lower the risk of disease and allergies. Human gut microbiota directly influences health and provides an extra means of adaptive potential to different lifestyles and varies with diet, lifestyle and environment. This has been shown in animal tests and also in human studies comparing the microbes of people with and without particular diseases [15]. Meanwhile, there is mounting evidence that babies born via caesarean section miss out on some of the microbes they would obtain 
through a vaginal birth, which make them more vulnerable to obesity, allergies and asthma. So how can one restore healthy gut microbiota, increase the good bacteria in the body, and give a boost to healthy living.

\section{Increase fibre intake}

Aim for more than $40 \mathrm{~g}$ per day, which is about double the current averages. Fibre intake has been shown to reduce heart disease and some cancers, as well as reduce weight gain. Eat as many types of fruit and veg as possible, and try to eat seasonally. The variety may be as important as the quantities, as the chemicals and types of fibre will vary, and each support different microbial species. Good examples are artichokes, leeks, onions and garlic, which all contain high levels of insulin (a prebiotic fibre). Some vegetables like lettuce have little fibre or nutrient value.

\section{Choose right food and drinks}

Mention 'bacteria' and memories of food poisoning and nasty infections probably spring to mind. Polyphenols are antioxidants that act as fuel for microbes. Examples are nuts, seeds, berries, olive oil, brassicas, coffee and tea - especially green tea. Also, try to increase intervals between meals to give your microbes a rest. Occasionally skip meals or have an extended fast - this seems to reduce weight gain.

Eat plenty of fermented foods containing live microbes [16, 17] Good choices are unsweetened yoghurt; kefir, which is a sour milk drink with five times as many microbes as yoghurt; raw milk cheeses; sauerkraut; kimchi, a Korean dish made from garlic, cabbage and chilli; and soybeanbased products such as soy sauce, tempeh and natto. In small quantities, alcohol has been shown to increase your gut diversity, but large amounts are harmful to your microbes and your health. Steer clear of artificial sweeteners like aspartame, sucralose and saccharine. These disrupt the metabolism of microbes and reduce gut diversity - in animal studies this has led to obesity and diabetes. Ditch the processed foods too, as these also upset microbes' metabolism.

\section{Avoid City pollution}

People living in rural areas have better microbes than city-dwellers. While you're at it, dust off your trowel: gardening and other outdoor activities are good for your microbiome.

\section{Keep pets}

Studies have shown that people living with dogs have more microbial diversity.

\section{Avoid antibiotics and non-essential medicine}

6 Antibiotics destroy good and bad microbes, and it can take weeks to recover, so don't take them unless you need them. Their use is also associated with obesity and allergies in animals. Even common medications like paracetamol and antacids can interfere with microbes.

7 Don't be hygiene obsessed

Fastidious washing and overuse of antibacterial sprays may not be good for your gut.

8 Spend time close to a lean person

Studies in mice have shown that leanness may be contagious. Microbes from a lean animal can reverse obesity in a fat one, but strangely, obesity microbes are harder to transmit than lean ones.

\section{Avoid food and vitamin supplements}

Only a tiny proportion of supplements have been shown to be beneficial. Instead, focus on eating a diverse range of real food to get all your nutrients.

\section{Skin}

Normal Skin microbiota: Skin is the body's largest organ and its first line of defence against microbiota even though it harbours between one million and one billion microorganisms per square $\mathrm{cm}$. The surface of the skin is never sterile even when it is clean; it is covered with a mixture of microbes called normal. Most of the time, these microbes do not cause illness and do not stimulate the immune system however if there is a break in the skin or if the immune system is weakened, then the microbes may cause a wound or skin infection.

In one survey of skin microbiota it is found that, Propionibacteria, Corynebacteria, and Staphylococcus spp. makes more than $62 \%$ of sequences detected at 20 body sites. Moist, oily, and dry regions were each associated with different levels of microbial species diversity, which was lowest in oily regions that were associated with the sebaceous glands. Using samples from all body locations, biological sex could be determined $80.0 \%$ of the time, which is greater than expected by chance. However, when biological sex was classified for each body location, the thigh could be correctly classified $100 \%$ of the time (19). One can therefore determine biological sex consistently based on the thigh region, but cannot classify sex with the same certainty using any other skin region.

The epidermis layer is relatively hostile to microbes because it is constantly shedding and also associated with antimicrobial compounds, low in moisture, high acidity, hydrophobicity, and salinity. Wounds are breaks in the integrity of the skin and tissues. They may be superficial cuts, scrapes or scratches but also include deeper cuts, punctures, burns, or may be the result of surgical or dental procedures.

\section{Superficial skin infections}

Superficial infections occur primarily in the outer layers of the skin but may extend deeper into the underlying (subcutaneous) lay [20].

Examples of bacteria that can cause skin infections include:

Bacteria that are often normally found on the skin (normal flora), such as species of Staphylococcus (staph) and Streptococcus (strep), are common causes.

Antibiotic-resistant bacteria, such as

Vibrio or Aeromonas species, often found in brackish water

Pseudomonas aeruginosa is associated with hot tubs.

Bacteroides and Clostridium species may cause deeper wound infections.

Tularemia - this infection is caused by Francisella tularensis bacteria. They primarily infect rabbits and rodents, but humans can get infections through tick or deer fly bites or contact with infected animals that can result in skin ulcers.

Anthrax - this is an infection caused by the bacteria Bacillus anthracis. It can infect the skin as well as the respiratory or digestive tracts. The bacteria live in the soil and primarily infect animals, such as cattle, deer, sheep and goats. Humans can be infected by handling the animals or their hair, hide, or meat.

Typical bacterial skin infections include $[19,20]$

Infected hair follicles (folliculitis)

Boils (furuncles)

Collection of boils involving several hair follicles and deeper layers of tissue (carbuncles)

Impetigo_-skin lesions and blisters (vesicles) 
Pressure sores (bed sores) and ulcers - these may be found in people who are immobilized or bedridden for long periods of time

Cellulitis - an infection often involving the subcutaneous and connective tissue of skin, causing redness, heat, and swelling

Necrotizing fasciitis - a serious but uncommon infection that can spread rapidly and destroy skin, fat, muscle tissue and fascia, the layer of tissue covering muscle groups. This type of infection often involves Group a streptococci, which are sometimes referred to as "flesh-eating bacteria."

Examples of common fungal skin infections include:

Ringworm, Athlete's foot, Yeast infections cause by Candida species may occur in the mouth (thrush) or on other moist areas of the skin,

Examples of viruses that cause skin infections include: 2 (HSV-1, HSV2) are responsible for a skin infection known as herpetic whitlow.

Skin and wound infections interfere with the healing process and can create additional tissue damage. They can affect anyone, but people with underlying conditions are at risk of slower wound healing and greater risk of wound infections. Examples of conditions that increase the risk of wound infections include:

Poor blood circulation, Diabetes, Weakened/suppressed immune system (e.g., HIV/AIDS, organ transplant recipient), Low mobility or immobility (e.g., confined to bed, paralysis) and Malnutrition

When infections penetrate deep into the body into tissues such as bone, or when they occur in tissue that has inadequate circulation, they can become difficult to treat and may become chronic infections. Many infections remain confined to a small area. Others may persist and, if untreated, become more severe and spread further and/or deeper into the body. Some infections spread to other organs and/or into the blood (septicemia) and cause a body-wide (systemic) infection

Regulatory factors: The main factors affecting the normal human skin microbiota are skin location on the body, gender, geographical location, ethnicity, skin (thick or thin), antibiotics used, and application of cosmetics, age, and health conditions [21]. Female skin microbial communities were significantly more diverse than males, and it may be due to physiological differences in hormonal secretions which would change the skin texture, $\mathrm{pH}$ etc [22]. Wound and skin infections trigger the body's immune system and cause inflamation and tissue damage within the skin.

\section{Female Reproductive Tract}

The definition of vaginal microbiota cannot be only related to the presence or absence of individual microorganisms even though; the vaginal microbiota is a compartment of the human microbiota [23]. The vaginal microbiota is considered as almost like a second immune system for women - it really protects women from all kinds of things, unique conditions of the vagina are characterized by a few microbial species, usually lactobacilli, which are able to utilize glycogen, which is under control of estrogens. Healthy women may be colonized with a nonlactobacillary microbiota whose (quality and quantity) is dependent on ethnicity of the woman. This microbiota is manifested by a low degree of diversity and by the high dynamics of changes of its composition under the influence of various exogenous and endogenous factors. Lactobacilli and other fermentative bacteria together with vaginal epithelial cells produce lactic acid and are responsible for acidifying vaginal milieu. Increase in diversity can be paradoxically associated with a dysbiosis such as bacterial vaginosis $[24,25]$. Individual species of lactobacilli mainly Lactobacillus crispatus characterize the main community state types in the vagina. Apart from lactobacilli, healthy women may be colonized with a non-lactobacillary microbiota whose rate is dependent again on ethnicity. Non culture-based modern technologies revealed a complex and dynamic system mainly dominated by several species of lactobacilli. The normal and the abnormal vaginal microbiota are complex ecosystems of more than 200 bacterial species influenced by genes, ethnic background and environmental and behavioural factors.

The qPCR diagnostic approach had a sensitivity and specificity of respectively $93 \%$ and $93 \%$ for Nugent-defined BV. The prevalence of BV defined by Nugent score was $21 \%$, whereas the prevalence of an abnormal vaginal microbiota was $28 \%$. High concentrations of Gardnerella vaginalis and/or Atopobium vaginae were found out by qPCR. Aerobic vaginitis seems to be an immunological disorder of the vagina with influence on the microbiota, which is here dominated by aerobic bacteria (Streptococcus agalactiae, Escherichia coli).

The composition of microbiota is important, but it is only one of the basic attributes of normal vaginal microbiota, but not sufficient; that is the functional definition of vaginal microbiota in relation to its structure and dynamics, including the influence of ethnicity, physiological status of the vagina, and genetic disposition of woman. including the influence of ethnicity; lactic acid; $\mathrm{pH}$; physiological status of the vagina; sexual hormones; sexual hormones historical and recent concept.; usually lactobacilli [26,27].

\section{During pregnancy}

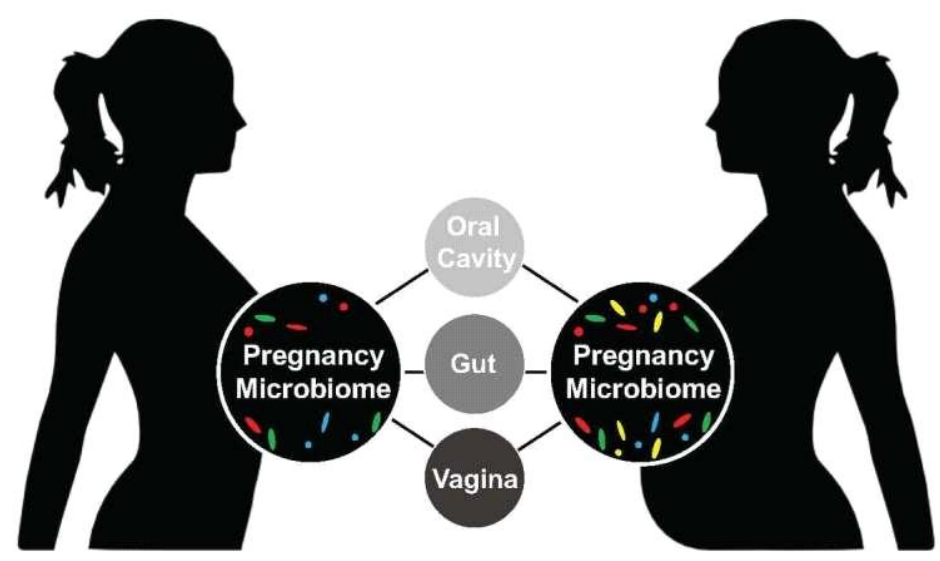

The human microbiota is dynamic during pregnancy, the microbial communities of three maternal body sites (vagina, oral cavity and skin) over the course of pregnancy from the first trimester through delivery shifts in strains, species and predicted functions at each colonized body site. Understanding its role in maternal health has important implications 
for fetal development and gestational outcome [28]. Yet, there remains a lack of detailed analyses regarding the structure, function, and temporal dynamics of the maternal microbiota and how it contributes to gestational outcome and fetal development $[29,30]$.

Researchers were surprised by the degree of diversity in the human microbiom at the level of bacterial strains and by their temporal patterns during the course of gestation. The vagina was primarily dominated by one organism and taxonomic diversity increased with gestational age. In the gut and oral cavity the communities displayed high diversity throughout gestation. Interestingly, the microbiota variation in the oral cavity was associated with pregnancy complications such as preeclampsia

$[31,32]$.

\section{Role to play by Microbiota}

\section{- Influence of the Microbiome on Infertility}

So far human infertility management personal never considered microbiota milieu as one of the parameters for infertile couples. Recent researches have shown the involvement of balanced and right composition of microbiota in gut, uterus, and vagina as well seminal fluid in man. Abnormal vaginal microbiota may negatively affect the clinical pregnancy rate in IVF patients. If a negative correlation between abnormal vaginal microbiota and the clinical pregnancy rate is corroborated, patients could be screened and subsequently treated for abnormal vaginal microbiota prior to fertility treatment. Although an altered vaginal microbiota has been demonstrated to affect parturition, its role in assisted reproductive technologies is uncertain. Nevertheless, the effect of known pathogens such as Mycoplasma tuberculosis, Chlamydia trachomatis, and Neisseria gonorrhoeae is clear, causing subclinical changes thought to be risk factors in subfertility. Studies that characterize the vaginal microbiota in assisted reproductive technology support the hypothesis that colonizing the transfer-catheter tip with Lactobacillus crispatus at the time of embryo transfer may increase the rates of implantation and live birth rate while decreasing the rate of infection. In addition, there is some evidence that a progesterone-resistant endometrium might increase the risk of an abnormal vaginal microbiome. It is also necessary to consider the differing racial and ethnic groups might have variations in what vaginal microbiota might be considered optimal in terms of health, it's becoming increasingly apparent that carrying Lactobacillus is more optimal than not and that Lactobacillus crispatus is particularly good. A phylogenic analysis of the vaginal microbiota indicated 4 bacterial species dominated: Lactobacillus iners, L. crispatus, L. gasseri, and L. jensenii. The lactic-acid producing bacteria, and particularly several species of Lactobacillus, are credited with that protective assist [33].

A dysbiotic microbiota composition may have an influence on sperm quality. In addition, we observed an increased relative abundance of Lactobacillus in samples with normal sperm morphology though; overall bacterial content of sperm might not play a major role in male infertility. Although no major shifts in microbiota composition or diversity were found, the differential abundance of specific bacterial genera in the sperm suggests that a small subset of microbes might impact the spermatozoal physiology during sperm transition, more specifically motility and morphology.

\section{- Epigenetic Effects}

Recent studies have demonstrated that epigenetic events are highly dynamic, changing in response to nutrient availability [34, 35]; the dynamic epigenetic modifications are carried out by enzymes such as DNA methyltransferases, DNA hydroxylases, histone acetyltransferases, histone deacetylases, histone methyltransferases, and histone demethylases. Krautkramer [36] Aleksandrova et al., [37] Romano et al
[38] have demonstrated that microbial colonization regulates global histone acetylation and methylation in multiple host tissues in a dietdependent manner.

Regulation of DNA and histone methylation may be driven by complex microbiota-host metabolism interactions involving S-adenosyl methionine (SAM), derived from the essential amino acid methionine through diet [38] Folatewhich is recommended during pregnancy, plays an essential role by re-methylating homocysteine to methionine.

Taken together, dysbiosis of microbiota can influence SAM levels and, as a result, alter the methylation status of DNA and histones. Whether dysbiosis of microbiota can alter $\alpha$-ketoglutarate and succinate levels in specific peripheral host tissues, and regulate the rate of DNA demethylation, is a plausible but little explored possibility. Histone phosphorylation is also regulated by microbial metabolism.

In short, dysbiosis and reduction of the microbiota diversity can potentially alter the levels of nutrients and metabolites that can potentially act as regulators of DNA methylation and histone modifications either by directly inhibiting enzymes that catalyze the processes, or by altering the availability of substrates necessary for the enzymatic reactions [39].

Yet another phenomenon in epigenetics is "Epigenetic Mosaicism" in which at the single-cell level, gene expression was highly variable and quite different than expected, which is now shedding light on the molecular causes of rare diseases and perhaps the complex nature of tumours. Epigenetics has many and varied potential in medical applications since, ultimately it turns out to have a greater role in prevention of diseases than classical genetics. Researchers so far have found epigenetic clues for management of not only to cancer, but also cardiovascular disease, diabetes, lupus and even autism [40].

\section{Perceptions of epigenetics.}

It was known that what we eat affects the microbes in our bodies. hundreds of different species of bacteria are present in the stomach, working to protect us as well as survive within us, however if due to change in diet if there is an imbalance in beneficial and pathogenic bacteria serious diseases can set in [40].

\section{a.How the Microbiome Affects Fertility}

Infertility is multifactorial (and sometimes more than one causes are involved) issues are involved however hither to unknown cause is gut "Microbiota". The prioritizing microbial health can positively impact a variety of common fertility roadblocks to encourage a happy outcome:a. Unfriendly Microbes Unwanted bacterial strains and yeast can affect a woman's ability to have a baby, whether couples are trying the old fashioned way or through IVF. Not only do these undesirable microbes negatively impact sperm, they can also make a woman's body less hospitable to her newly conceived child. Supporting the gut microbiota helps crowd out harmful microbes throughout the body, including those that may impact fertility.

Introducing probiotics directly to the vaginal area may also be helpful. An IVF study involving healthy women showed promising results after vaginal colonization with L. crispatus. And research with mice found that intravaginal application of L. plantarumim proved the chances of successful reproduction, even when certain troublesome strains were also added to the mix [41].

\section{b. Temporary Inflammation}

When temporary inflammation occurs due to irritation, injury, or unfriendly microbes, it may affect a woman's cycles and immune system, which can make it more difficult to conceive and carry a baby.5 A number of probiotic strains are associated with supporting immune function though, and some also show potential for supporting healthy 
fertility. Research involving 33,000 Norwegian women found that supplementing with milk-based probiotics reduced temporary inflammation and some associated pregnancy issues [42].

\section{Sperm Health}

The more densely populated with sperm a man's semen is, and the faster those little guys can swim, the better his odds of contributing his DNA to a brand new life. And it turns out the composition of semen microbiome actually affects sperm vitality [43].

One study involving couples who had been unable to conceive revealed that when friendly Lactobacillus dominates the semen microbiome, the sperm is much more likely to be healthy and normal. Additional research with mice found that taking the probiotic L. reuteri orally increased sperm count and testicular mass, as well as kept aging reproductive organs healthy.

\section{Excess Weight}

Carrying more than your optimal body weight can compromise health in many ways, not the least of which is a negative impact on both male and female fertility. For men, those unwanted pounds may change levels of testosterone and other hormones, which ultimately harm sperm health. Extra weight in women can also lead to hormonal changes that affect her cycles, as well as whether or not pregnancy progresses smoothly [44, 45].

But what's a couple to do if they've tried every diet and exercise plan imaginable and the pounds still aren't coming off? Recent research reveals that the gut microbiome is an important factor in healthy weight management. So, something as simple as introducing friendly probiotics to the digestive tract can make it easier for your body to manage weight for healthy fertility.

\section{Health}

Both externally and internally human tissues and body fluids are shelter homes for microbiota. The composition of the aggregates of biota is not the same; this depends on the type of tissue and/or fluid. These microorganisms are not harmful to humans; in fact, some are even beneficial and all help maintain our health. However if species balance in their population is disturbs, we face serious concequences [46, 47].

These microbes help digest food we eat, set our metabolism rate, regulate our weight and moderate our immune system. But in recent years, scientists have wondered whether the wrong balance of microbial populations - brought about by an unhealthy, Western-style diet might be responsible, at least in part, for the rise in some modern chronic diseases, such as obesity and irritable bowel syndrome. So, a group of scientists led by microbiologist Justin Sonnenburg of Stanford University decided to study the gut microbiome in its most natural, preindustrial setting: by examining the Hadza people of Tanzania.

The Hadza comprise one of the last groups of humans living a traditional, nomadic, hunter-gatherer lifestyle, as all humans once did just a few tens of thousands of years ago. Despite having no access to modern health care, the Hadza are largely free from the chronic diseases that plague Americans.

What the scientists found is that the Hadza have a far more diverse gut microbiome compared to Americans. What's more, the types of gut bacteria vary greatly in number as the Hadza alter their diet from season to season.

The new study, published Aug. 252017 in the journal Science [48], suggests that a person's diet strongly dictates the diversity of the gut microbiome, and that those people living in the industrialized world have a far less vibrant gut microbiome that may adversely affect their health.

Effect of Common Medicines on Microbiota
A new study shows that some widely used drugs alter the population of microbes in the gut that includes at least 1,000 species of bacteria and is influenced by a number of different factors, including medication., which in turn raise the risk of antibiotic resistance, [49] It is already know that the efficiency and the toxicity of certain drugs are influenced by the bacterial composition of the gastrointestinal tract and that the gut microbiota has been related to multiple health conditions; therefore, it is crucial to understand what are the consequences of medication use in the gut microbiome.

The gut microbiomes of PPI users had higher levels of upper gastrointestinal tract bacteria and increased fatty acid production, and metformin users had higher levels of potentially harmful E. coli bacteria.

Seven other drug categories were associated with significant changes in bacterial populations in the gut, according to the researchers.

For example, the use of SSRI anti - depressants by people with IBS was associated with increased levels of the potentially harmful bacteria species Eubacterium ramulus.

Meanwhile, the use of oral steroids was associated with high levels of methanogenic bacteria linked with obesity and an increase in body mass index (an estimate of body fat based on weight and height).

The importance of considering the role of the gut microbiota associated with obesity, diabetes, liver diseases, cancer and neurodegenerative diseases when designing treatments and also points to new hypotheses that could explain certain side-effects associated with medication use.

\section{What Does Your Microbiome Say About You?}

It wasfound that personality traits were significantly correlated with diversity of gut microbiota, High neuroticism and low conscientiousness groups were correlated with high abundance of Gammaproteobacteria and Proteobacteria, respectively when covariates, including age, sex, BMI and nutrient intake, were controlled. Additionally, high conscientiousness group also showed increased abundance of some universal butyrateproducing bacteria including Lachnospiraceae. This study was of observational and cross-sectional design and our findings must be further validated through metagenomic or metatranscriptomic methodologies, or metabolomics-based analyses. Our findings will contribute to elucidating potential links between the gut microbiota and personality, and provide useful insights toward developing and testing personality- and microbiota-based interventions for promoting health [50].

\section{Conclusion}

Different areas of the body host characteristic microbiota. There are more microbial cells in the body than the body cells itself, and cumulatively they weigh 1.4 to $2.3 \mathrm{~kg}$. The myriad microorganisms living in/on the human body is not a new discovery. Initially it was thought they are parasites only but now their new role was discovered. Actually they are essential component of our life. The relationship between humans and bacteria is much more complicated, however. We feed the bacteria as we eat, the bacteria in turn churn out stuff that is both wanted and unwanted in our gut. Different strains produce different stuff. Some can be beneficial, some are toxic. The human microbiota is studied in relation to health or disease, these microorganisms play a role in all sorts of diseases, and even mental health problems As long as the strains of microbiota stay in 'balance', they counter-check each other, so that none of the strains overgrow and cause problems. It's when that equilibrium is disrupted that things go wrong. When the balance is upset, they can cause many diseases including cancer. 'Overuse and underuse' of antibiotics is one of the cause of microbiota upset.

A newborn's microbes are compromised when they are delivered via Csection or fed formula. Mothers should opt for vaginal delivery and 
breastfeeding for healthy newborn. Maternal gut microbiota is important because mothers pass on their gut microbiota to their infant in various ways (direct transfer, potentially in breast milk), and her gut microbiota may also influence the development of the metabolic and immune systems of the fetus during gestation - in utero programming.

\section{References}

1. louis-pasteur, Apr 27, 2017

2. Brummer, et al. (2018) The role of the microbiome for human health: from basic science to clinical applications Nutr.57 (Suppl 1): 1-14.

3. Ding T, Schloss PD. (2014) Dynamics and associations of microbial community types across the human body. Nature 509: 357-360.

4. Sender R, Fuchs S, Milo R. (2016) Revised Estimates for the Number of Human and Bacteria Cells in the Body. PLoS Biol 14(8): e1002533.

5. Sprockett D, Fukami T, Relman DA. (2018) Role of priority effects in the early-life assembly of the gut microbiota. Nat Rev Gastroenterol Hepatol; 15:197-205.

6. The Human Microbiome Project Consortium. (2012) Structure, function and diversity of the healthy human microbiome. Nature, 486, 207-214.

7. Milani C, Duranti S, Bottacini F, et al. (2017) The first microbial colonizers of the human gut: composition, activities, and health implications of the infant gut microbiota. Microbiol Mol Biol Rev; 81(4):e00036-17.

8. Maurice, C. F. \& Turnbaugh, P. J. (2013) Quantifying and identifying the active and damaged subsets of indigenous microbial communities. Methods Enzym. 531, 91-107.

9. Huse SM, Ye Y, Zhou Y, Fodor AA. (2012) A Core Human Microbiome as Viewed through 16S rRNA Sequence Clusters. PLoS ONE 7(6): e34242.

10. Knight R, Petrosino JF, et al. (2012) The Human Microbiome Project: a community resource for the healthy human microbiome. PLoS Biol. 10(8):e1001377.

11. Seigle-Murandi F, Guiraud P, et al. (1996) Bacteria Are Omnipresent on Phanerochaete chrysosporium Burdsall. Appl Environ Microbiol. ; 62(7):2477-81.

12. Milani C., Ferrario C.,et al. (2016) The human gut microbiota and its interactive connections to diet. J Hum Nutr Diet 29, 539-5.

13. wen L , Andrew Duffy (2017) Factors Influencing the Gut microbiota, Inflammation, and Type 2 Diabetes , J Nutrition, 147,(7), 1468S-1475S.

14. Carmody, R.N., Bisanz, J.E., Bowen, B.P. et al. (2019) Cooking shapes the structure and function of the gut microbiome. Nat Microbiol. 4(12) :2052-2063.

15. David, L. A. et al. (2014) Dietrapidly and reproducibly alters the human gut microbiome Nature 505, 559-563.

16. A Ashley. Kirsten M. Ross, et al. (2018) Comprehensive skin microbiome analysis reveals the uniqueness of human skin and evidence for phylosymbiosis within the class Mammalia. Proc Natl AcadSci. USA 115 (25) E5786-E5795.

17. Noah Fierer, Christian L. Lauber, Nick Zhou, Daniel McDonald, Elizabeth K. (2010) Costello, and Rob Knight Forensic identification using skin bacterial communities Proc Natl AcadSci U S A. 107(14): 6477-6481.

18. Chen YE, Fischbach MA, Belkaid Y. (2018) Skin microbiotahost interactions.Nature. 553(7689):427-436.

19. Byrd AL, Belkaid Y, Segre JA. (2018) The human skin microbiome. Nat Rev Microbiol. 16(3):143-155.
20. Boskey ER, Cone RA, Whaley KJ, Moench TR. (2001) Origins of vaginal acidity: High D/L lactate ratio is consistent with bacteria being the primary source. Hum Reprod16: 1809-1813.

21. Younes JA, Lievens E, Hummelen R, van der Westen R, Reid G, Petrova MI. (2018) Women and Their Microbes: The Unexpected Friendship. Trends Microbiol. ; 26(1):16-32.

22. P.D. Gupta (2021) the Human Vaginal Microbiota: Boon or Bane. J of Obstetrics Gynecology and Reproductive Sciences $5(1)$

23. Gonçalves LF, Chaiworapongsa $T$, Romero R. (2002) Intrauterine infection and prematurity. Ment Retard Dev Disabil Res Rev 8: 3-13 25

24. Boskey ER, Cone RA, et al. (2001) Origin of vaginal acidity: High D/L lactate ratio is consistent with bacteria being the primary source. Hum Reprod16: 18090-1813.

25. Boskey ER, Telsch KM, Whaley KJ, Moench TR, Cone RA. (1999) Acid production by vaginal flora in vitro is consistent with the rate, extent of vaginal acidification. Infect Immun 67: 5170-175.

26. Chelsea Fox. (2015) Maternal microbiome and pregnancy outcomes Fertility and Sterility 104, (6), 1358-1363.

27. Verstraelen,H., Verhelst,R., et al. (2009) tudinal analysis of the vaginal microflora in pregnancy suggests that $\mathrm{L}$. cripatus promotes the stability of the normal vaginal microflora and that L. gasseri and/or L. iners are more conducive to the occurrence of abnormal vaginal microflora. BMC Microbiology, 9, 116.

28. Versalovic, J. (2012) A metagenomic approach to characterization of the vaginal microbiome signature in pregnancy. PLOS ONE, e36466.

29. Walther-António, M. R. S., Jeraldo, P., Breg Miller,M. E., Yeoman, C. J.,Nelson, K. E., Wilson, B. A., \& Creedon, D. J. (2014) egnancy'sstronghold on the vaginal microbiome. PLOS ONE, 9, e98514.

30. PD Gupta and K Pushkala. (2020) Hidden Reasons of Woman's Infertility: Microbiota Milieu Acta Scientific Women's Health $2(2)$

31. Jacques Ravel, Pawel Gajer, et al. (2011) Vaginal microbiome of reproductive-age women. Proc Natl Acad Sci , 108 (Supplement 1) 4680-4687.

32. Bird A. (2007) Perceptions of epigenetics. Nature 447: 396398.

33. K Pushkala, P D Gupta. (2013) Epigenetic Effect of food for cancer management. ,Intl J Med Sci Biotech 1 (II)

34. Krautkramer KA, Kreznar JH, et al. (2016) Diet-Microbiota Interactions Mediate Global Epigenetic Programming in Multiple Host Tissues. Mol Cell. ; 64(5):982-992.

35. Aleksandrova K, Romero-Mosquera B, Hernandez V. (2017) Diet, Gut Microbiome and Epigenetics: Emerging Links with Inflammatory Bowel Diseases and Prospects for Management and Prevention. Nutrients. ; 9(9):962.

36. Romano,Kymberleigh A CampoAna Martinez-del, et al. (2017) Metabolic, Epigenetic, and Transgenerational Effects of Gut Bacterial Choline Consumption Cell Host \& Microbe 22, 279_ 290.

37. El Hajj N, Schneider E, Lehnen H, Haaf T. (2014) Epigenetics and life-long consequences of an adverse nutritional and diabetic intrauterine environment. Reproduction.148 (6):R11120.

38. Tycko B. (2003) Genetic and epigenetic mosaicism in cancer precursor tissues. Ann N Y Acad Sci. 983:43-54.

39. Koedooder R, Singer M, et al. (2019) The vaginal microbiome as a predictor for outcome of in vitro fertilization with or without intracytoplasmic sperm injection: a prospective study. Hum Reprod. 34(6):1042-1054. 
40. Suzie Sorenson Fertility and the Microbiome: How Probiotics Can Help You Conceive

41. Poutahidis, T., Springer, A., et al. (2014) Probiotic Microbes Sustain Youthful Serum Testosterone Levels and Testicular Size in Aging Mice. PLoS ONE, 9(1), e84877.

42. Leddy MA, Power ML, Schulkin J. (2008) The impact of maternal obesity on maternal and fetal health. Rev Obstet Gynecol.; 1(4):170-8.

43. Vidal, A. C.,Murphy, S. K.,Murtha, A. P., Schildkraut, J.M., Soubry, A., Huang, Z., \& Hoyo, C. (2013) Associations between antibiotic exposure during pregnancy, birth weight and aberrant methylation at imprinted genes among offspring. International Journal of Obesity, 37, 907-913.
44. Neiger R. (2017) Long-Term Effects of Pregnancy Complications on Maternal Health: A Review. J Clin Med. 6(8):76.

45. Moloney, R. D., Desbonnet, L., et al. (2014) The microbiome: Stress, health and disease. Mammalian Genome, 25, 49-74.

46. Smits S A Leach J et al. (2017) Seasonal cycling in the gut microbiome of the Hadza hunter-gatherers of Tanzania, Science. 357, (6353), 802-806.

47. Gupta, P. D Bacteria: (2018) The Powerful Creatures: A Mini Review. J Cell Tissue Res 18(3) 6555-6558.

48. Cryan JF, Dinan TG. (2012) Mind-altering microorganisms: the impact of the gut microbiota on brain and behaviour. Nat Rev Neurosci; 13:701-12.

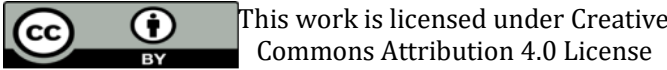

To Submit Your Article Click Here: Submit Manuscript

DOI: $10.31579 / 2693-4779 / 048$
Ready to submit your research? Choose Auctores and benefit from:

* fast, convenient online submission

* rigorous peer review by experienced research in your field

* rapid publication on acceptance

* authors retain copyrights

* unique DOI for all articles

* immediate, unrestricted online access

At Auctores, research is always in progress.

Learn more www.auctoresonline.org/journals/clinical-research-andclinical-trials 